this turns out to be a co-operative process: at intermediate stages of the reaction only intact unreacted and fully reacted dissociated species were present. The rate-determining factor is then the reactivity of the native enzyme (most probably in fact its dissociation into the reactive sub-units) and this can be measured. Addition of the two substrate species, succinate and carbamyl phosphate, led to a large increase in the rate of reaction, which was partly annulled by the inhibitor CTP. The substrates at the same time brought about a small diminution in sedimentation coefficient, which was again opposed by CTP. From the absence of any dependence on enzyme concentration, it was surmised that the substrates induce a true change in frictional coefficient rather than a gross shift in dissociation equilibrium. It does therefore appear that an appreciable structural change occurs when the substrates are attached. Changeux and Rubin (ibid., 561) show that the experimental data can be satisfactorily fitted on the basis of the twostate allosteric model, such that the substrate binds only to one of the conformational isomers, the inhibitor preferentially to the other, and that in the absence of ligands the two states co-exist, with a four-fold preponderance of the catalytically active form.

It is worth noting, as part of the discussion of allosterie mechanisms, an article by Sweeny and Foster (ibid., 561) in which a clear formulation is given of the generation of sigmoid binding curves by isolated catalytic sites. The requirement is only that there shall be alternative reaction pathways to the proteinligand complex. Several such models are described. Another interesting analysis of co-operative binding is given by Klapper and Klotz (ibid., 223) for situations involving an aggregation equilibrium in which the affinities of the associated and dissociated states for a given ligand differ. Measurement of ligand affinities can be used to yield the equilibrium constant for the protein dissociation, and a striking application to the dissociation of haemerythrin is described.

\section{Symmetry of RNA Transcription}

\section{from our Cell Biology Correspondent}

IN 1966, Szybalski and his collaborators proposed that clusters of pyrimidine residues in DNA are somehow related to RNA transcription, possibly acting as initiation and termination signals for RNA polymerase. At the time the evidence for this hypothesis was thin, and the theory itself may well be an oversimplification, but all the evidence obtained since is compatible with Szybalski's suggestion. In the latest paper from Szybalski's laboratory, Summers and Szybalski (Virology, 34,$9 ; 1968$ ) report that more than 99.8 per cent of the $T_{7}$ and more than 99 per cent of the $T_{3}$ coliphage messenger RNA molecules that are made throughout the phage growth cycle are transcribed from only one of the two complementary strands of $T_{7}$ and $T_{3}$ DNA. And the strand which is transcribed contains clusters of cytosine residues, while the strand that is not transcribed has neither cytosine nor thymine clusters.

Summers and Szybalski used the DNA/RNA hybridization technique to study $T_{7}$ and $T_{3}$ transcription and the success of their experiments depended on their ability to separate the two complementary strands of $T_{7}$ and $T_{3}$ DNA. They did this with the techniques that have been developed by Szybalski's group over the last three years for separating the DNA strands of phage and bacteria. If one of the two strands of a DNA molecula contains more cytosine than the other, they can be fractionated by first heating the DNA to separate the strands, then binding a polyribonucleotide rich in guanine to the $C$ rich strands, so increasing its density, and then separating it from the $C$ poor strand in a caesium chloride gradient.

Having separated $T_{7}$ and $T_{3}$ DNA strands in this way, Summers and Szybalski were able to show that all the messenger RNA made at both early and late times hybridizes specifically with the $C$ cluster strand. Control experiments proved that the two DNA strands are not self-complementary and that $C$ poor strand is available for hybridization so that its failure to hybridize $m$ RNA is not an artifact.

Clearly only one strand of $T_{7}$ and $T_{3}$ DNAs, the one rich in cytosine elusters, acts as template for messenger RNA. Similarly, Mamur and Greenspan (1963) reported that only the pyrimidine rich strand of phage $S P 8$ hybridizes with SP8 mRNA. Szybalski's group (Taylor, Hradneca and Szybalski, Proc. US Nat. Acad. Sci., 5\%, 1619; 1967) reported that both strands of $\lambda$ DNA act as templates and this correlates well with the occurrence of pyrimidine rich clusters on both strands. Also Habich et al. (1966) found that although both strands of Bacillus megatherium DNA act as template for $m \mathrm{RNA}$, the pyrimidine rich strand contains most templates. And $m$ RNA must also be transeribed off both strands of $T_{4}$ DNA because Streisinger et al. (J. Mol. Biol., 31, 607; 1968) have concluded from genetic experiments that the lysozyme and $r$ II genes are translated in the opposite direction to $T_{4}$ head protein.

Given the correlation between template function of DNA strands and their high content of pyrimidine clusters, it is tempting to speculate that $d C$ or $d T$ clusters act as initiation and possibly termination signals for RNA polymerase. Two in vitro experiments with RNA polymerase support this speculation. First, most $m$ RNA made in vitro with $E$. coli as templates has $A$ or $G$ at the $5^{\prime}$ terminus (Maitra and Hurwitz, 1965). Second, Chamberlain (1967) reported that when poly $d C d G$ or poly $d T d A$ are used as templates in vitro, RNA initiation and termination occur very frequently.

What sort of signals can clusters of pyrimidines provide? Langridge (1967) found that synthetic double helical DNA with pyrimidines on one strand and purines on the other had slightly different structure from native DNA. Summers and Szybalski suggest that this modified structure in the regions of native DNA with pyrimidine clusters could serve as a recognition region for RNA polymerase.

\section{Isoenzymes and Cancer}

\section{from our Medical Biochemistry Correspondent}

IT is now well known that lactate dehydrogenase (LDH) is found in five electrophoretically separable forms known as isoenzymes or isozymes. The enzyme molecule is made up of four sub-units, each of which can be one of two types known as $H$ and $M$ sub-units. The possible combinations of $H$ and $M$ sub-units give the five forms of LDH which can be separated by electrophoresis in suitable conditions.

In a study of the LDH isozymes in normal human uterus, in myoma and in carcinoma of the uterine. 